\title{
Single-bubble dynamics in pool boiling of one-component fluids
}

\author{
Xinpeng $\mathrm{Xu}^{1}$ and Tiezheng Qian ${ }^{2, *}$ \\ ${ }^{1}$ Department of Mathematics, Hong Kong University of Science and Technology, Clear Water Bay, Kowloon, Hong Kong \\ ${ }^{2}$ Department of Mathematics and KAUST-HKUST Micro/Nanofluidics Joint Laboratory, Hong Kong University of Science and Technology, \\ Clear Water Bay, Kowloon, Hong Kong
}

(Received 27 January 2014; published 4 June 2014)

\begin{abstract}
We numerically investigate the pool boiling of one-component fluids with a focus on the effects of surface wettability on the single-bubble dynamics. We employed the dynamic van der Waals theory [Phys. Rev. E 75, 036304 (2007)], a diffuse-interface model for liquid-vapor flows involving liquid-vapor transition in nonuniform temperature fields. We first perform simulations for bubbles on homogeneous surfaces. We find that an increase in either the contact angle or the surface superheating can enhance the bubble spreading over the heating surface and increase the bubble departure diameter as well and therefore facilitate the transition into film boiling. We then examine the dynamics of bubbles on patterned surfaces, which incorporate the advantages of both hydrophobic and hydrophilic surfaces. The central hydrophobic region increases the thermodynamic probability of bubble nucleation while the surrounding hydrophilic region hinders the continuous bubble spreading by pinning the contact line at the hydrophobic-hydrophilic intersection. This leads to a small bubble departure diameter and therefore prevents the transition from nucleate boiling into film boiling. With the bubble nucleation probability increased and the bubble departure facilitated, the efficiency of heat transfer on such patterned surfaces is highly enhanced, as observed experimentally [Int. J. Heat Mass Transfer 57, 733 (2013)]. In addition, the stick-slip motion of contact line on patterned surfaces is demonstrated in one-component fluids, with the effect weakened by surface superheating.
\end{abstract}

DOI: 10.1103/PhysRevE.89.063002

PACS number(s): 47.55.D-, 68.08.-p, 68.03.Fg, 44.35.+c

\section{INTRODUCTION}

Boiling is commonly observed in our daily life and is ubiquitous in many geophysical and astrophysical systems [1-3]. Technically, boiling is recognized as a highly efficient mode of heat transfer that has been widely used in a variety of applications in, for example, chemical engineering, the power industry, the steel industry, and the microelectronics industry $[1,2]$.

Physically, boiling is an extremely complex and elusive nonequilibrium process, in which various physical components are involved and interrelated [1-4], e.g., nucleation, growth, and departure of vapor bubbles; latent heat transport accompanying the liquid-vapor transition; hydrodynamic instability of the liquid-vapor interface; and hydrodynamic interactions between bubbles. This complexity in physics and the technical advantage of efficient heat transfer have attracted extensive research efforts from both scientists and engineers. Early in the second half of the 20th century, boiling heat transfer had been studied extensively, largely stimulated by the industrial exploitation of high-power heat exchangers in nuclear power stations $[5,6]$. Up to now, the qualitative nature of boiling has been well documented for most of the common fluids and common regimes [1-3]. Quantitatively, numerous correlations of boiling heat transfer have been presented empirically involving several adjustable parameters [1-3]. Below is a summary of a few key aspects of pool boiling, i.e., boiling on a heating surface submerged in a pool of initially quiescent liquids [1-3].

(i) Boiling regimes. Phenomenologically, pool boiling with controlled surface temperature can be divided into three

*Corresponding author: maqian@ust.hk distinct regimes: nucleate boiling, transition boiling, and film boiling.

(ii) Superheated liquid layer and nucleate boiling regime. When the temperature of the heating surface is slightly above the saturation temperature of the liquid, the liquid layer near the heating surface is superheated and nucleate boiling takes place, characterized by the nucleation and growth of vapor bubbles [7] and their departure driven by the buoyant forces and surface tension [4]. The bubble dynamics induces the microconvection in the superheated liquid layer and leads to a very large rate of heat transfer from the solid to the fluid.

(iii) Transition boiling regime. As the temperature of the heating surface is increased above a threshold, continuous vapor films are formed over portions of the solid surface. Since vapor is less capable of conducting heat, these vapor films essentially insulate the bulk liquid from the heating surface, resulting in a dramatic decrease in the rate of heat transfer. Moreover, these vapor films are unstable and can detach from the surface. This restores the contact of liquid with solid and resumes the nucleate boiling. This regime, which combines unstable film boiling with partial nucleate boiling, is usually referred to as the regime of transition boiling.

(iv) Critical heat flux. The critical heat flux (CHF) is a characteristic heat flux that marks the transition from the nucleate boiling to the transition boiling. In the nucleate boiling regime, the heat flux increases with increasing surface temperature while in the transition boiling regime, the heat flux decreases with increasing surface temperature as the heating surface becomes more and more covered by vapor films. In industrial practices with controlled surface heat flux, the performance of boiling equipments is generally limited by the $\mathrm{CHF}$, characterized by a rapid increase of surface temperature. This results in the physical "burnout" of the heating surface, the "boiling crisis." 
(v) Leidenfrost temperature and film boiling regime. When the surface temperature is further increased above the socalled Leidenfrost temperature, the liquid layer near the heating surface becomes thermodynamically unstable and film boiling takes place, characterized by the presence of a stable vapor film insulating the liquid from the solid. The rate of heat transfer reaches a minimum at the Leidenfrost temperature.

(vi) Effects of surface wettability. The boiling dynamics and the rate of heat transfer are greatly affected by the surface wettability. In general, when the degree of surface superheating and the liquid pressure are given, a higher heat flux would be obtained if the liquid wets the surface better. Moreover, the above three boiling regimes are identified specifically for partial wetting. If the liquid does not wet the heating surface, then the boiling process immediately enters into the film boiling regime as soon as boiling is initiated. The nucleate boiling regime, the $\mathrm{CHF}$, and the transition boiling regime become unobservable.

Although the boiling phenomena have been extensively studied for decades, most studies are empirical in nature and only a few studies have focused on the fundamental understanding of the boiling process, leaving it virtually unexplored in physics [3,5,6,8-10]. Below we list a few problems posed by the boiling process with a focus on its thermohydrodynamic aspects.

(i) Most of the questions to be answered are closely related to the boiling dynamics in the close vicinity of the heating surface, in particular, the bubble dynamics down to the contact-line scale. On the one hand, there are difficulties in the experimental observation and theoretical analysis of the complicated, violent boiling process. On the other hand, the complexity of the boiling process and the well-known stress and thermal singularities associated with the contact line hugely complicate direct numerical simulations.

(ii) The mechanism underlying the boiling crisis remains controversial [5,11-13]. The boiling crisis occurs at the CHF, marking the transition from nucleate boiling to transition boiling and eventually to film boiling. Two major mechanisms have been proposed. The first one argues that the bubble crowding close to the heating surface leads to the fusion of multiple dry spots. The other considers surface drying initiated by the growth of a single bubble.

It has been the belief of many people that numerical simulations of the complete boiling process may be the only viable option [3,6]. Recently, there have been various attempts to simulate boiling numerically. In general, there exist three different methods.

(i) Continuum method. Extended thermohydrodynamic models. The classical thermohydrodynamics [14] can be extended to liquid-vapor flows on heating surfaces by directly solving the Navier-Stokes equation and various conservation laws of relevant physical quantities in the liquid and vapor phases, respectively. This must be supplemented with a model for the liquid-vapor interfaces and necessary boundary conditions at the solid surface [15]. For decades, there have been numerous works for the continuum modeling and simulations of boiling $[6,16,17]$. However, most of these works made use of empirical models for the liquid-vapor interfaces, with ad hoc assumptions on the contact line dynamics. (ii) Mesoscopic method. Lattice Boltzmann method (LBM). The LBM is a popular mesoscopic method for the simulation of two-phase flows on solid substrates [18-20]. Recently, many works have been carried out to simulate the boiling process by extending the LBM to liquid-vapor flows that involve the liquid-vapor transition in nonuniform temperature fields [21-23]. However, the validity of these works still need further deliberation.

(iii) Molecular method. Molecular dynamics (MD) simulation. MD simulation is one of the most promising methods to reproduce boiling numerically. An MD model deals with a system consisting of a number of particles interacting with each other. By choosing a few reasonable interactions between the particles and using proper boundary conditions, the boiling of a liquid can be reproduced in a natural and comprehensive manner without introducing numerical artifacts [24]. Recently, there have been extensive studies of processes related to boiling using MD simulations [24-28]. However, these simulations are limited to systems of very small size (up to a micrometer).

Recently, the dynamic van der Waals theory (DVDWT) has been presented for one-component two-phase hydrodynamics involving liquid-vapor transition in nonuniform temperature fields $[29,30]$. As a diffuse-interface model formulated for liquid-vapor flows, the DVDWT provides an alternative and effective continuum method for investigating the thermohydrodynamics of boiling process down to the contact line scale in the close vicinity of the heating substrate. Supplemented with the hydrodynamic boundary conditions at the fluid-solid interface [31], this model is able to fully take into account the various physical processes involved in the contact line dynamics, including the liquid-vapor transition (evaporation or condensation) and capillary flow in the bulk fluid region, and boundary slip of fluid at solid surface [31]. Due to the use of the diffuse-interface method, the stress and thermal singularities at the contact line are resolved automatically [31]. Furthermore, the DVDWT treats the evaporation or condensation rate at the liquid-vapor interface as an outcome of calculation rather than a prerequisite as in most of the existing continuum models for liquid-vapor flows involving liquid-vapor transition [31]. With the help of these advantages, the DVDWT has been employed to study the evaporation, spreading, and migration of evaporative droplets on solid substrates, from the complete wetting regime to the partial wetting regime and to the nonwetting (Leidenfrost) regime [28,31-35].

It is interesting and important to note that the DVDWT has recently been used to study the pool boiling of one-component fluids [36]. However, the competition between lateral bubble spreading and vertical bubble departure, which is an important characteristic of pool boiling under gravity, has not been addressed and systematically investigated. The outcome of this competition is essential to the triggering of boiling crisis and to the transition from nucleate boiling to film boiling regime $[12,13]$. One of the main purposes of the present work is to employ the DVDWT to investigate the competition between lateral bubble spreading and vertical bubble departure in saturated pool boiling. The DVDWT is numerically implemented by using an explicit finite difference scheme. Numerical results are obtained for the growth and departure of bubbles on homogeneous and chemically patterned heating surfaces, respectively. 
The paper is organized as follows. In Sec. II, we briefly review the DVDWT with the boundary conditions and describe its numerical implementation. In Sec. III, we study the growth and departure of a single bubble on homogeneous surface under zero gravity and artificially large gravity, respectively. Simulations for zero gravity are motivated by the initial stage of bubble growth, in which the bubble size is so small that the role of gravity is negligible. Simulations under artificially large gravity are numerical experiments to reproduce the buoyantinduced bubble departure. In Sec. IV, we investigate the bubble dynamics on chemically patterned surface, motivated by the large rate of heat transfer measured in a recent experiment [37]. We find that the stick-slip motion of contact line leads to a reduced departure diameter of bubbles, which is partly responsible for the enhanced rate of heat transfer. We conclude the paper in Sec. V with a few remarks.

\section{THEORETICAL MODELING AND NUMERICAL IMPLEMENTATION}

We consider the pool boiling of a one-component fluid on a heating solid substrate. In the framework of the DVDWT [30,31], we take into account a number of physical components involved and interrelated in the boiling process: two-phase hydrodynamics, liquid-vapor transition, latent-heat transport, and wetting dynamics at solid surface. Assuming the solid substrate to be flat, rigid, and of high heat conductivity, we outline a minimal version of the DVDWT below $[30,31,38]$.

\section{A. Dynamic van der Waals theory}

The DVDWT is formulated for heterogeneous onecomponent fluids with coexisting liquid and vapor. In this diffuse interface model, the natural order parameter is the number density $n$, which varies quickly but smoothly across liquidvapor interfaces. The entropy density $n s$ for homogeneous fluids is generalized by including a gradient contribution, taking the form of

$$
\hat{S}=n s(n, e)-\frac{C}{2}|\nabla n|^{2},
$$

where $s(n, e)$ is the entropy per molecule for homogeneous fluids of number density $n$ and internal energy density $e$. For simplicity, we assume $C$ to be a positive constant that results in a decrease of entropy due to the density inhomogeneity $[30,31,33,39]$. In this case, the entropy and internal energy are respectively given by $S_{b}=\int d \mathbf{r} \hat{S}$ and $E_{b}=\int d \mathbf{r} e$. We then define the temperature $T$ via

$$
\frac{1}{T} \equiv\left(\frac{\delta S_{b}}{\delta e}\right)_{n}=n\left(\frac{\partial s}{\partial e}\right)_{n}
$$

and the generalized chemical potential $\hat{\mu}$ via

$$
\hat{\mu} \equiv-T\left(\frac{\delta S_{b}}{\delta n}\right)_{e}=\mu-C T \nabla^{2} n,
$$

in which $\mu=-T[\partial(n s) / \partial n]_{e}$ is the chemical potential for homogeneous fluids.

In the van der Waals theory [10,39], homogeneous onecomponent fluids are characterized by two fundamental parameters: the molecular volume $v_{0}$ and the strength of attractive interaction $\varepsilon$. The Helmholtz free energy density $f(n, T)$ is given by $f(n, T)=n k_{B} T\left[\ln \left(\lambda_{\mathrm{th}}^{3} n\right)-1-\ln \left(1-v_{0} n\right)\right]-$ $\varepsilon v_{0} n^{2}$, from which the entropy per molecule $s$, the internal energy density $e$, and the pressure $p$ (equation of state) can be obtained as

$$
\begin{gathered}
s(n, T)=k_{B} \ln \left[\left(k_{B} T / \varepsilon\right)^{3 / 2}\left(1 / n v_{0}-1\right)\right]+\text { const, } \\
e(n, T)=3 n k_{B} T / 2-\varepsilon v_{0} n^{2}, \\
p(n, T)=n k_{B} T /\left(1-v_{0} n\right)-\varepsilon v_{0} n^{2} .
\end{gathered}
$$

Here $\lambda_{\text {th }} \equiv \hbar\left(2 \pi / m k_{B} T\right)^{1 / 2}$ is the thermal de Broglie wavelength with $k_{B}, m$, and $\hbar$ being the Boltzmann constant, molecular mass, and Planck constant, respectively. Note that $s(n, e)$ can be readily derived from Eq. (4) and Eq. (5). Moreover, the critical density, temperature, and pressure are respectively given by

$$
n_{c}=1 / 3 v_{0}, \quad T_{c}=8 \varepsilon / 27 k_{B}, \quad p_{c}=\varepsilon / 27 v_{0} .
$$

To model the wettability effects of solid surface, we introduce the surface entropy $S_{s}=\int d A \sigma_{s}(n)$ and the surface energy $E_{s}=\int d A e_{s}(n)$, in which $d A$ is the surface element, and the areal densities $\sigma_{s}$ and $e_{s}$ are assumed to only depend on the fluid density $n$ at the solid surface. It follows that the equilibrium conditions can be obtained by maximizing the total entropy $S_{\text {tot }} \equiv S_{b}+S_{s}$ subject to fixed particle number $N=\int d \mathbf{r} n$ and fixed total internal energy $E_{\text {tot }} \equiv E_{b}+E_{s}$. They read (i) $T=$ const in the whole system, (ii) $\hat{\mu}=\mathrm{const}$ in the bulk region, and (iii)

$$
C T \nabla_{\gamma} n+\left(\partial f_{s} / \partial n\right)_{T}=0
$$

at the solid surface. Here $\nabla_{\gamma} \equiv \hat{\gamma} \cdot \nabla$ with $\hat{\gamma}$ denoting the outward unit vector normal to the surface and $f_{s}(n, T) \equiv$ $e_{s}(n)-T \sigma_{s}(n)$ is the surface free energy per unit area. Note that due to the introduction of a gradient contribution in Eq. (1), a length scale $\ell$ can defined by

$$
\ell=\left(C / 2 k_{B} v_{0}\right)^{1 / 2},
$$

which is the characteristic liquid-vapor interfacial thickness far from the critical point. In addition, from the equilibrium condition $\hat{\mu}=$ const, an estimate of the liquid-vapor interfacial tension $\gamma$ is obtained as [38]

$$
\gamma \sim k_{B} T\left(1-T / T_{c}\right)^{3 / 2} \ell / v_{0} .
$$

Now we present the hydrodynamic equations from the conservation laws of mass and momentum plus the principle of positive entropy production in irreversible processes. The mass density $\rho \equiv m n$, the momentum density $\rho \mathbf{v}$, and the entropy density $\hat{S}$ satisfy the balance equations

$$
\begin{gathered}
\frac{\partial n}{\partial t}+\nabla \cdot(n \mathbf{v})=0, \\
\frac{\partial}{\partial t}(\rho \mathbf{v})+\nabla \cdot(\rho \mathbf{v v})=\nabla \cdot(-\overleftrightarrow{\Pi}+\overleftrightarrow{\boldsymbol{\sigma}})-\rho g \mathbf{e}_{z}, \\
\frac{\partial \hat{S}}{\partial t}+\nabla \cdot(\hat{S} \mathbf{v})=-\nabla \cdot \hat{\mathbf{J}}_{f}^{S}+\sigma .
\end{gathered}
$$



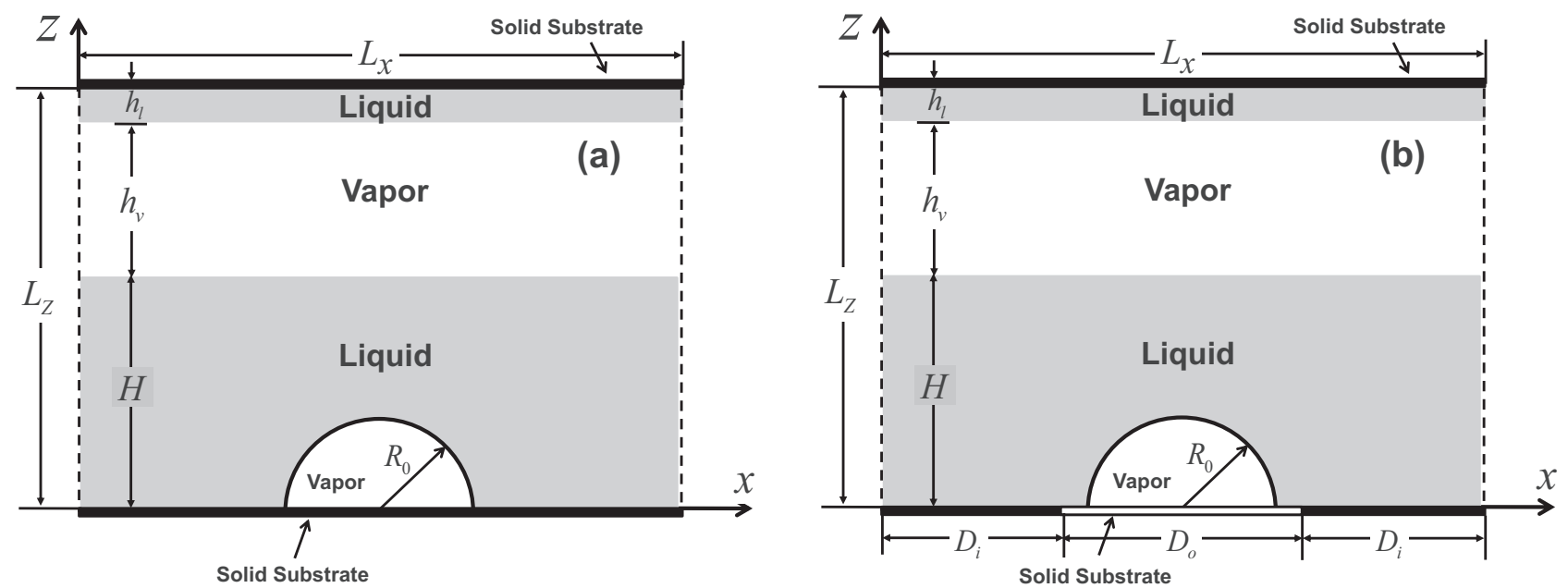

FIG. 1. Schematic illustration for simulated systems. (a) A bubble on a homogeneous surface, to be discussed in Sec. III. (b) A bubble on a chemically patterned (hydrophilic-hydrophobic-hydrophilic) surface, to be discussed in Sec. IV. The black and white segments of the solid surface indicate the hydrophilic and hydrophobic parts, respectively. The system is closed in the $x$ direction by the periodic boundary conditions. Note that a vapor layer of thickness $h_{v}$, sandwiched by liquids in the $z$ direction, is introduced to maintain the supersaturated state of the liquid surrounding the bubble. This is essential to the simulation of boiling processes $[30,36]$.

In Eq. (12), $g$ is the gravitational acceleration and $\mathbf{e}_{z}$ is the (upward) unit vector along the $z$ axis (see Fig. 1). The total stress tensor is divided into two parts. The Newtonian viscous stress tensor $\overleftrightarrow{\boldsymbol{\sigma}}$ is given by

$$
\overleftrightarrow{\boldsymbol{\sigma}}=\eta\left(\nabla \mathbf{v}+\nabla \mathbf{v}^{T}\right)+(\zeta-2 \eta / 3) \overleftrightarrow{\mathbf{I}} \nabla \cdot \mathbf{v}
$$

with $\eta$ and $\zeta$ being the shear and bulk viscosities, respectively, and the generalized reversible pressure tensor $\overleftrightarrow{\boldsymbol{\Pi}}$ (with gradient contribution) is given by

$$
\stackrel{\leftrightarrow}{\boldsymbol{\Pi}}=\left[p-C T\left(n \nabla^{2} n+|\nabla n|^{2} / 2\right)\right] \overleftrightarrow{\mathbf{I}}+C T \nabla n \nabla n,
$$

where $p$ is expressed by Eq. (6) and the anisotropic part $-C T \nabla n \nabla n$ results in the liquid-vapor interfacial tension $\gamma$. In Eq. (13), the generalized entropy flux $\hat{\mathbf{J}}_{f}^{S}$ (with gradient contribution) is given by

$$
\hat{\mathbf{J}}_{f}^{S}=-\frac{1}{T} \lambda \nabla T-C n(\nabla \cdot \mathbf{v}) \nabla n,
$$

with $\lambda$ being the heat conductivity, and the entropy production rate in the bulk region $\sigma$ is given by

$$
\sigma=\frac{1}{T} \overleftrightarrow{\boldsymbol{\sigma}}: \nabla \mathbf{v}+\frac{1}{T^{2}} \lambda(\nabla T)^{2}
$$

in the linear response regime.

In order to use the above system of equations to simulate the pool boiling process, we need appropriate boundary conditions at the heating surface. For simplicity, we work under the following assumptions:

(i) For fluid velocity $\mathbf{v}$, the no-slip boundary condition $\mathbf{v}=\mathbf{0}$ is applied.

(ii) For fluid temperature $T$, the isothermal (Dirichlet) boundary condition $T=$ const is applied. This means we consider the boiling processes under the condition of controlled surface temperature. (iii) For number density $n$, the equilibrium condition in Eq. (8), i.e., $C T \nabla_{\gamma} n+\left(\partial f_{s} / \partial n\right)_{T}=0$, is applied. This means the equilibration at the solid boundary is much faster than that in the bulk.

It is interesting to note that molecular dynamics simulations have shown that (i) fluid velocity slip becomes appreciable in the vicinity of a moving contact line $[40,41]$ and (ii) a jump of temperature (due to the Kapitza resistance) can occur across the fluid-solid interface, especially for liquids on hydrophobic substrates [42,43]. In addition, the heat conductivity of the substrate can be finite in comparison with that of the liquid, and the equilibrium condition in Eq. (8) can be relaxed for slow equilibration at the solid surface [32,41]. These effects are to be described by a more general set of boundary conditions, which have been derived in our recent work [31].

In summary, we will simulate the pool boiling process by numerically solving Eqs. (11)-(13) supplemented with appropriate boundary conditions and initial conditions. Our simulations will provide detailed thermohydrodynamic information of the boiling process down to the contact-line scale. This will be achieved by analyzing the evolution of the density distribution $n(\mathbf{r}, t)$ (and, hence, the bubble profile) calculated from Eq. (11), the velocity distribution $\mathbf{v}(\mathbf{r}, t)$ calculated from Eq. (12), and the temperature distribution $T(\mathbf{r}, t)$ calculated from Eq. (13) together with Eqs. (1), (4), and (5). Here the entropy equation is used instead of the energy equation in order to avoid the numerical parasitic flows $[30,44]$.

\section{B. Numerical method and system setup}

The above system is numerically solved by using an explicit finite-difference scheme [30,31,33,38]. Numerical simulations are carried out in the two-dimensional $x z$ plane with the fluids confined in the computational domain $0 \leqslant x \leqslant L_{x}$ and $0 \leqslant z \leqslant L_{z}$, as illustrated in Fig. 1 and displayed in Figs. 2 and 6 . The state variables $n, \mathbf{v}$, and $T$ in the fluid are defined in a two-dimensional, unstaggered, uniformly 

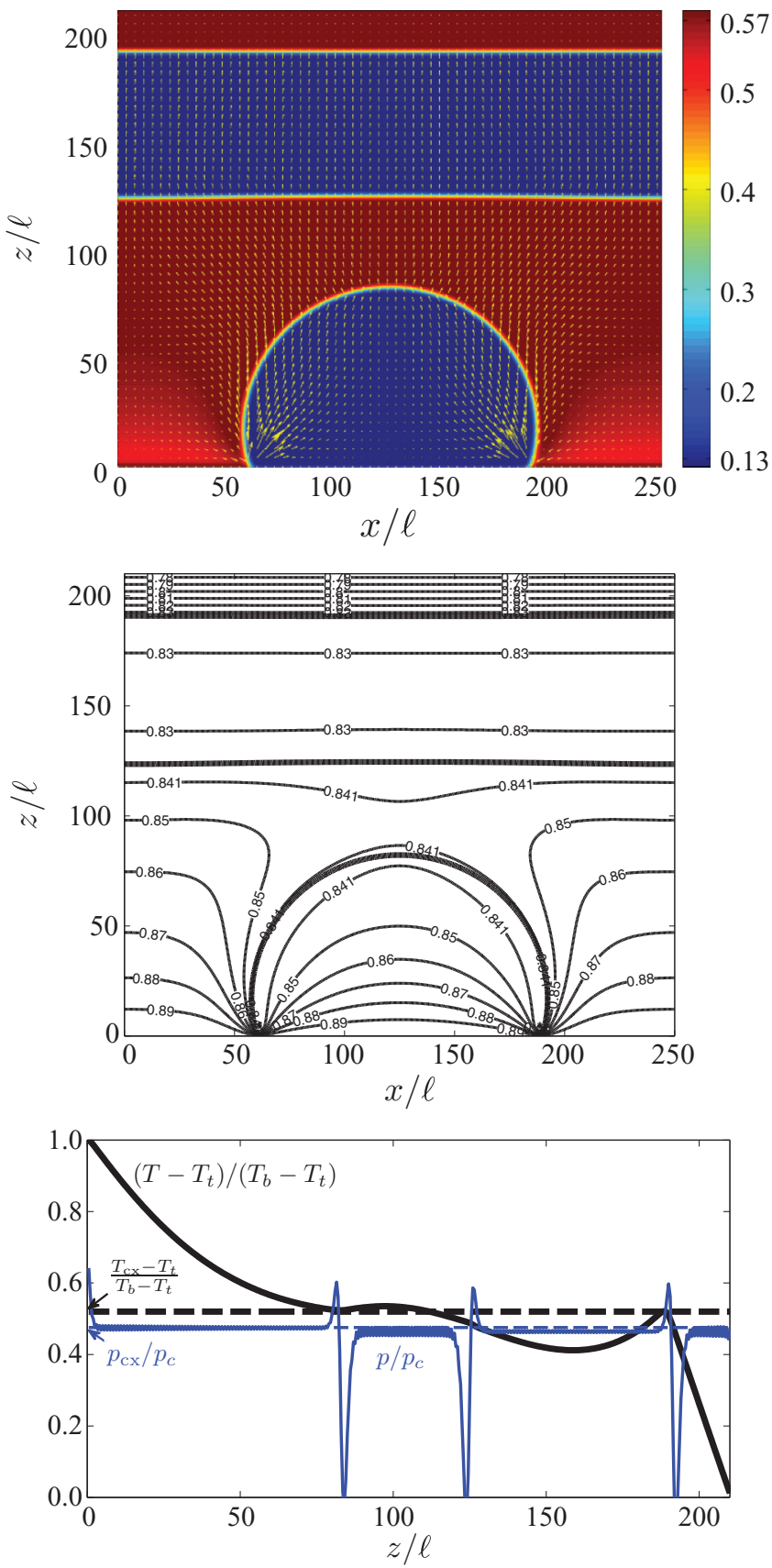

FIG. 2. (Color online) A bubble under zero gravity on a homogeneous surface with static contact angle $\theta_{s}=0^{\circ}$ and surface temperatures $T_{b}=0.90 T_{c}$ and $T_{t}=0.775 T_{c}$, recorded at time $t=5000 \tau_{0}$. The system measures $L_{x}=250 \ell$ by $L_{z}=210 \ell$ with $h_{l}=10 \ell$, $h_{v}=100 \ell$, and $H=100 \ell$ as defined in Fig. 1 . Upper panel: Density (color) and velocity (arrow) fields. Middle panel: Temperature contour (labeled thin lines) and liquid-vapor interfaces defined at $n=\left(n_{g}+n_{l}\right) / 2$ (thick lines). Temperature values are in units of $T_{c}$. Lower panel: Reduced pressure $p / p_{c}$ with $p$ calculated from Eq. (6) (thin solid blue line) and reduced temperature $\left(T-T_{t}\right) /\left(T_{b}-T_{t}\right)$ along the $z$ direction at $x=125 \ell$ (thick solid black line). The thin dashed blue line denotes the coexistence pressure $p_{\mathrm{cx}} \approx 0.48 p_{c}$, and the thick dashed black line denotes the coexistence temperature $T_{\mathrm{cx}} \approx 0.84 T_{c}$.

discretized Cartesian mesh. The mesh size is chosen to be $\Delta x=\Delta z=0.5 \ell$ with $\ell$ defined in Eq. (9). The two solid surfaces are defined at $z=0$ and $L_{z}$, and periodic boundary conditions are applied in the $x$ direction to close the system. In this geometry, the isothermal (Dirichlet) boundary condition for temperature is given by $T=T_{b}$ at the bottom surface ( $z=0)$ and $T=T_{t}$ at the top surface $\left(z=L_{z}\right)$, where $T_{b}$ and $T_{t}$ are given constants. As illustrated in Fig. 1, a thick vapor layer, sandwiched by liquids in the $z$ direction, is introduced. This is to maintain the supersaturated state of the liquid surrounding the bubble. Physically, this metastable state is a key factor in simulating the boiling dynamics [30,36]. Alternatively, an open boundary condition may be applied at the top of the surrounding liquid ( $z=H$ here) [36].

To further simplify our simulations, we assume $e_{s}=$ const, $\sigma_{s}=-c_{s}\left(n-n_{c}\right)$ with $c_{s}=$ const. Hence, the surface free energy density is given by

$$
f_{s}=c_{s} T\left(n-n_{c}\right)+\text { const. }
$$

Commonly used in the study of wetting phenomena [45], this kind of surface energy function leads to density enrichment or depletion near the solid surface. The viscosities $\eta$ and $\zeta$ and the heat conductivity $\lambda$ are assumed to be locally proportional to $n$, i.e.,

$$
\eta=\zeta=v m n, \quad \lambda=v k_{B} n,
$$

with the kinematic viscosity $v$ being a constant.

In our computation, the number density is measured by $1 / v_{0}$, the space by the characteristic interfacial thickness $\ell$, the time by the viscous relaxation time $\tau_{0} \equiv \ell^{2} / \nu$, the velocity by $V_{0} \equiv \ell / \tau_{0}=v / \ell$, and the temperature by the critical temperature $T_{c}$. By dedimensionalizing the hydrodynamic equations and boundary conditions, we obtain three dimensionless parameters [33], $\mathcal{R} \equiv v^{2} m / \varepsilon \ell^{2}$, which characterizes the dynamic properties of the bulk fluid,

$$
\mathcal{G} \equiv m g \ell / \epsilon,
$$

which is the normalized gravitational acceleration, and $\mathcal{W} \equiv$ $c_{S} / k_{B} \ell$ for the wettability of the solid surface. In this work, we use $\mathcal{R}=0.06$ and vary $\mathcal{W}$ to simulate different wettabilities (i.e., different static contact angles $\theta_{s}$ ).

We want to point out that the DVDWT is employed to simulate the bubble dynamics with a small set of material parameters: the molecular mass $m$, the energy parameter $\varepsilon$, the volume parameter $v_{0}$, the coefficient $C$ for the gradient contribution to entropy density, and the kinematic viscosity $\nu$. All the other material parameters are expressed in terms of these five basic parameters. Physically, the use of $\mathcal{R}=0.06$ can be justified by a simple argument on the sound propagation in viscous fluids [30]. In one-component fluids, the sound velocity $c$ scales as $(v / \ell) \mathcal{R}^{-1 / 2}$ and the acoustic oscillation becomes apparent for small $\mathcal{R}$ : $\mathcal{R} \ll(\ell q)^{-2}$, where $q$ is the wave number. For wavelengths as short as $\ell$ (with $q \sim 1 / \ell$ ), we have $\mathcal{R} \ll 1$. In addition, we require that the acoustic traversal time $L_{z} / c$ should be much longer than $\tau_{0} \equiv \ell^{2} / \nu$, the microscopic viscous relaxation time on the scale of $\ell$ (the smallest time scale in our simulations). With $L_{z} \sim 10^{2} \ell$ in our simulations, we obtain $\mathcal{R} \gg\left(\ell / L_{z}\right)^{2} \sim 10^{-4}$. It is interesting and necessary to investigate the effect of viscosity on bubble dynamics, for which prior studies have been carried out $[3,46-48]$. 
The normalized gravitational acceleration $\mathcal{G}$ needs a particular explanation [35]. By definition, $\mathcal{G}$ measures the gravitational energy of a molecule over the distance $\ell$ relative to the interaction energy $\varepsilon$. For example, $\mathcal{G} \approx 1.6 \times 10^{-14}$ for water, which is obtained for $\ell \approx 1 \mathrm{~nm}$ and $g=9.8 \mathrm{~m} / \mathrm{s}^{2}$. Physically, $\mathcal{G}$ is directly related to the capillary length defined by $R_{c} \equiv\left(\gamma / n_{l} m g\right)^{1 / 2}$ with $n_{l}$ being the liquid number density. It represents the length scale at which the gravitational energy and the liquid-vapor interfacial energy are comparable. In the pool boiling scenario, the bubble dynamics on the heating surface is influenced by the gravity only if the size of the growing bubble becomes comparable with $R_{c}$. At the coexistence temperature $T_{\mathrm{cx}}=0.84 T_{c}$ with $n_{l} \approx 0.61 / v_{0}, R_{c}$ is estimated to be $R_{c} \approx 0.16 \ell / \mathcal{G}^{1 / 2}$ from $T_{c}=8 \varepsilon / 27 k_{B}$ and Eqs. (10) and (20). For water under the Earth's gravity, $\mathcal{G} \approx$ $1.6 \times 10^{-14}$ gives the capillary length $R_{c} \approx 1.1 \mathrm{~mm}$, which is a macroscopic length scale, larger than $\ell(\approx 1 \mathrm{~nm})$ by six orders of magnitude. Numerically, our simulations are limited to nanoscale systems (hundreds of $\ell$ in each direction), in which the Earth's gravity is not relevant. Therefore, in order to induce appreciable gravitational effects in our simulations, we have to use artificially large gravity by taking $\mathcal{G}=10^{-4}$ and $\mathcal{G}=5.0 \times 10^{-5}$, which give $R_{c} \approx 16 \ell$ and $R_{c} \approx 23 \ell$, respectively. This allows us to investigate the gravitational effects on bubble dynamics near the heating surface. It is worth emphasizing that the use of artificially large gravitational acceleration is, on the one hand, necessitated by the multiscale nature of the problem and, on the other hand, caused by our limited computational capability.

Our simulations start from the equilibrium state of twophase coexistence at temperature $T_{\mathrm{cx}} \approx 0.84 T_{c}$, pressure $p_{\mathrm{cx}} \approx$ $0.48 p_{c}$, and densities $n_{l} \approx 0.61 / v_{0}$ and $n_{v} \approx 0.10 / v_{0}$ for liquid and vapor, respectively. The top-surface temperature is then changed to $T_{t}=0.775 T_{c}$, and a semicircular vapor bubble of radius $R_{0}(=25 \ell$ or $20 \ell$ ) is introduced at the bottom surface. This is realized by locally changing the fluid density from $n_{l}$ to $n_{v}$ in a prescribed region (of bubble shape). The purpose of this operation is to mimic the generation of vapor bubble via thermodynamic nucleation. It is noted that there exists a short transient of fast bubble relaxation ( $\sim$ hundreds of $\tau_{0}$ ) mainly caused by the mismatch between $R_{0}$ and the surrounding thermal and mechanical conditions. The wettability of heating surface, manifested via the static contact angle, is adjusted by varying the dimensionless parameter $\mathcal{W} \equiv c_{s} / k_{B} \ell$, which takes the values $-0.07,-0.04,-0.02,0.02,0.06$, and 0.09 , corresponding to static contact angles $\theta_{s}=180^{\circ}, \theta_{s}=120^{\circ}$, $\theta_{s}=101^{\circ}, 76^{\circ}, 41^{\circ}$, and $0^{\circ}$, respectively. (Here the contact angle is defined in the liquid phase.) The degree of surface superheating, defined by $\Delta T_{\text {sup }} \equiv T_{b}-T_{\text {cx }}$, is changed by fixing the coexistence temperature $T_{\mathrm{cx}} \approx 0.84 T_{c}$ and varying the bottom-surface temperature $T_{b}$ from $0.88 T_{c}$ to $0.93 T_{c}$. Finally, the total flux of heat transfer from the bottom surface at $z=0$ to the fluid is computed from

$$
Q_{b}=-\int_{0}^{L_{x}} d x(\lambda \partial T / \partial z)_{z=0}
$$

which is usually taken as a measure of the efficiency of boiling heat transfer [1-3,30]. Physically, heat transfer in pool boiling is carried by conduction, convection, and latent-heat transport accompanying the liquid-to-vapor transition.

\section{BUBBLE GROWTH AND DEPARTURE ON HOMOGENEOUS SURFACES}

In the nucleate boiling regime, the liquid layer superheated near the surface experiences large density fluctuations. As a result, vapor bubbles are nucleated at active sites of the heating surface. And when they reach a critical radius, $R_{n}$, they grow spontaneously. Typically, $R_{n}$ is very small, of the order of magnitude of hundreds of angstroms [1-3]. The growth of the vapor bubbles proceeds in three successive stages [1-3]:

(i) In the initial stage, the bubble size is so small that the growth proceeds continuously in the superheated liquid [7]. Here the gravity is negligible. Moreover, the speed of lateral bubble spreading over the heating surface is not only determined by the rate of bubble growth (depending on the degree of surface superheating) but also controlled by the degree of surface wettability.

(ii) When the bubble size becomes comparable with the capillary length $R_{c}$, the gravity (buoyancy) induces the vertical bubble deformation, which competes with the lateral spreading.

(iii) With further increase of the bubble size, the vertical bubble deformation becomes dominant, leading to the bubble departure from the heating surface eventually. In subcooled boiling, the detached bubble gradually shrinks and finally collapses in the subcooled liquid in the bulk. In saturated boiling, however, the detached bubble grows continuously during its rise in the saturated liquid. This will be the focus of the present work.

In this section, we present our hydrodynamic simulations for the above three stages of bubble growth in saturated pool boiling. We first perform simulations under zero gravity, motivated by the initial stage of bubble growth, in which the bubble is very small compared to the capillary length. We then simulate the growth and departure of nanoscale bubbles under artificially large gravity. This is for a numerical demonstration of the dynamics of large bubbles $(\sim 1 \mathrm{~mm})$ under the Earth's gravity.

\section{A. Bubble growth under zero gravity}

Consider a bubble on a homogeneous surface under zero gravity. In order to investigate the effects of surface wettability on the growing dynamics of a single bubble, we vary the static contact angle $\theta_{s}$, which is controlled by the dimensionless parameter $\mathcal{W} \equiv c_{s} / k_{B} \ell$.

Starting from the equilibrium state of two-phase coexistence at temperature $T_{\mathrm{cx}} \approx 0.84 T_{c}$, we introduce a single bubble of initial radius $R_{0}=25 \ell$ on the bottom surface at $t=0$. The temperatures of the bottom and top surfaces are then changed to be $T_{b}=0.90 T_{c}$ and $T_{t}=0.775 T_{c}$, respectively. In Fig. 2, we plot the number density $n$, velocity $\mathbf{v}$, temperature $T$, and pressure $p$ for a representative state. Here the static contact angle is $\theta_{s}=0^{\circ}$. The upper panel of Fig. 2 shows that the dynamic contact angle is much larger than its static value $\theta_{s}=0^{\circ}$ due to the significant surface superheating $[13,44]$. The liquid-to-vapor transition is localized in the close vicinity 

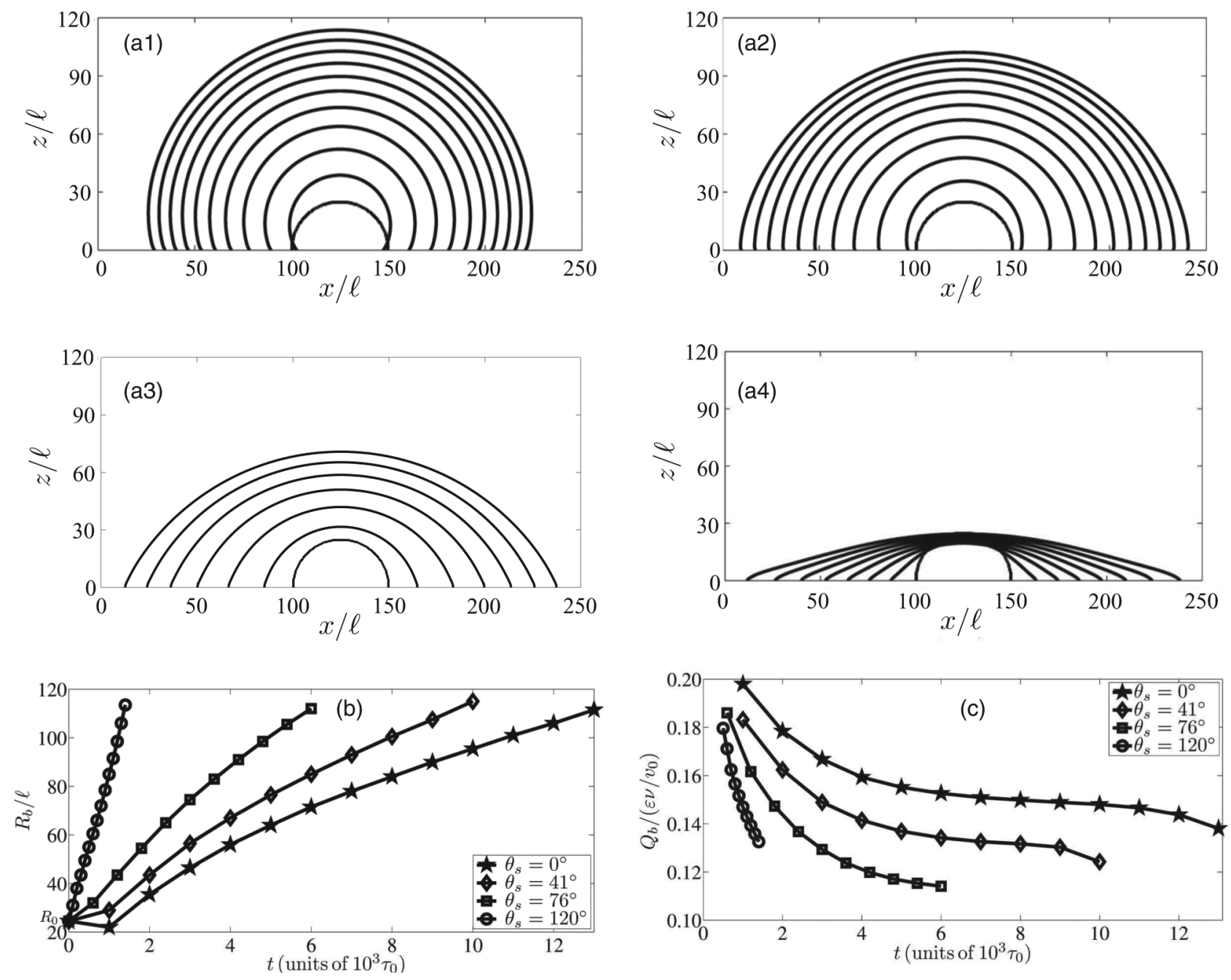

FIG. 3. Bubble growth on homogeneous surfaces under zero gravity; effects of surface wettability. (a) Temporal evolution of a bubble on a surface with a given static contact angle $\theta_{s}$ : (a1) $\theta_{s}=0^{\circ}$, (a2) $\theta_{s}=41^{\circ}$, (a3) $\theta_{s}=76^{\circ}$, and (a4) $\theta_{s}=120^{\circ}$. The time step is $\Delta t=1000 \tau_{0}$ in (a1)-(a3) and $\Delta t=200 \tau_{0}$ in (a4). (b) Temporal evolution of the bubble base radius $R_{b}$ (half the length over which the bubble is in contact with the solid) in units of $\ell$. (c) Temporal evolution of the surface-integrated heat flux $Q_{b}$ (in units of $\epsilon \mathcal{V} / v_{0}$ ), computed from Eq. (21). Here the system measures $L_{x}=250 \ell$ by $L_{z}=210 \ell$ with $h_{l}=10 \ell, h_{v}=100 \ell$, and $H=100 \ell$ as defined in Fig. 1 . The surface temperatures are $T_{b}=0.90 T_{c}$ and $T_{t}=0.775 T_{c}$, respectively.

of the contact line over the length scale of interfacial thickness $(\approx 3 \ell)[33,34]$. The middle panel of Fig. 2 shows that the temperature of the liquid-vapor interfaces is everywhere close to the coexistence temperature $T_{\mathrm{cx}} \approx 0.84 T_{c}$, a characteristic of one-component fluids. This indicates the presence of a thermal singularity at the contact line, characterized by a very large temperature gradient and thus a very large heat flux $[33,44]$. These properties associated with the liquid-vaporsolid three phase contact line in one-component fluids have been well studied for evaporative droplets on heating surfaces $[33,34,38,44]$. Furthermore, the middle panel of Fig. 2 shows that the thermal diffusion layer extends up to the top surface of the bubble. This is attributed to the small system size [10,30]. The pressure (shown in the lower panel of Fig. 2) outside the bubble is almost constant, close to the coexistence pressure $p_{\mathrm{cx}} \approx 0.48 p_{c}$. (Note that the sharp variation of $p$ in the liquid-vapor interfacial region is simply caused by the density variation therein [30].) The pressure inside the bubble is sightly higher than outside by $\gamma / R \approx 0.01 p_{c}$, implying that mechanical equilibrium is achieved quickly in such extremely nonisothermal environment [49]. This ensures a constant-pressure boiling condition [36].

Figures 3(a1)-3(a4) show the evolution of bubble profile on the heating surface. It is interesting to note that the shape of a growing bubble remains fairly circular, indicating that mechanical equilibrium is quickly achieved, already shown in the lower panel of Fig. 2. Therefore, the time dependence of the liquid-vapor interfacial curvature radius can be used to deduce that of the pressure inside the bubble.

Figures 3(b) and 3(c) show the effects of surface wettability on the temporal evolution of the bubble base radius $R_{b}$ (half the length over which the bubble is in contact with the solid) and that of the heat flux $Q_{b}$ computed from Eq. (21). The former gives the speed of lateral bubble spreading while the later measures the efficiency of heat transfer. We find that the larger the static contact angle $\theta_{s}$, the faster the bubble 

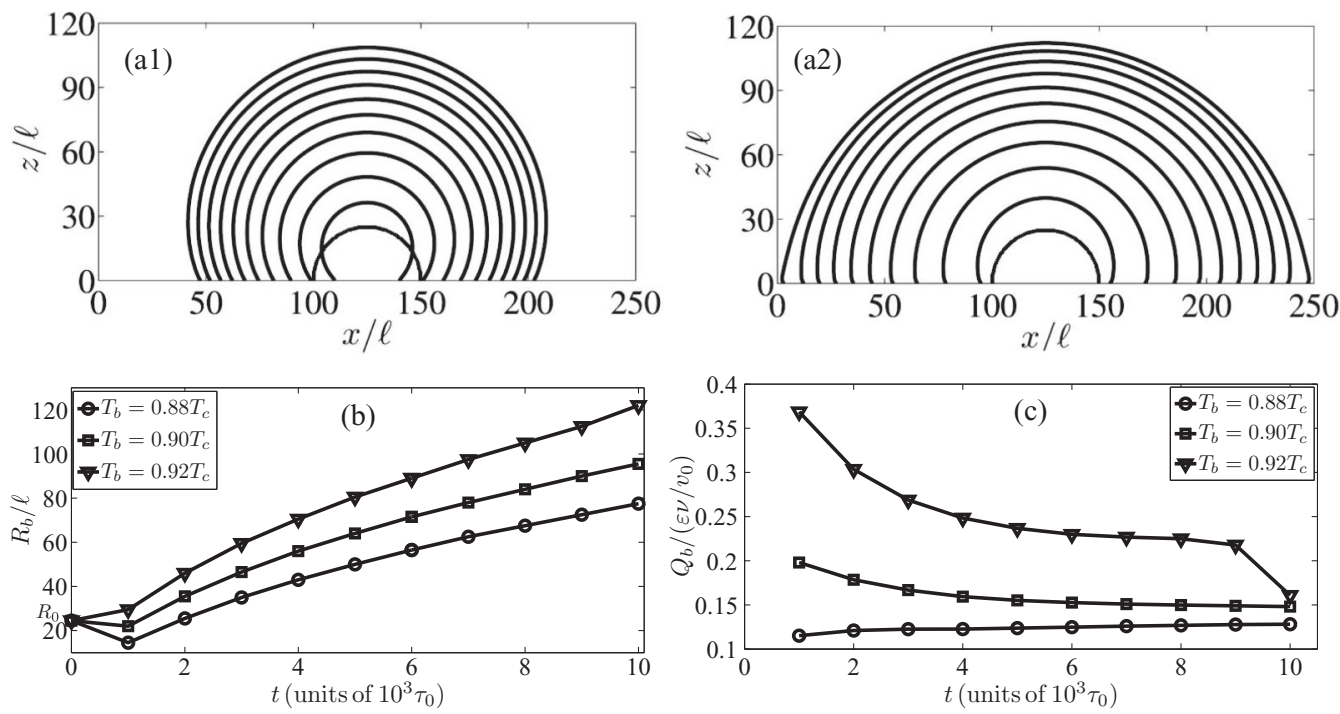

FIG. 4. Bubble growth on homogeneous surfaces under zero gravity; effects of surface superheating $\Delta T_{\text {sup }} \equiv T_{b}-T_{\text {cx }}$. (a) Temporal evolution of a bubble on a surfaces with a given bottom-surface temperature: (a1) $T_{b}=0.88 T_{c}$ and (a2) $T_{b}=0.92 T_{c}$. The top-surface temperature is $T_{t}=0.775 T_{c}$. The time step is $\Delta t=1000 \tau_{0}$. (b) Temporal evolution of the bubble base radius $R_{b}$ in units of $\ell$. (c) Temporal evolution of the surface-integrated heat flux $Q_{b}$ (in units of $\epsilon \mathcal{V} / v_{0}$ ), computed from Eq. (21). Here the system measures $L_{x}=250 \ell$ by $L_{z}=210 \ell$ with $h_{l}=10 \ell, h_{v}=100 \ell$, and $H=100 \ell$ as defined in Fig. 1. The static contact angle of the bottom surface is $\theta_{s}=0^{\circ}$.

spreads laterally on the heating surface and, consequently, the faster the heat transfer efficiency drops. This correlation is not surprising because the heat conductivity of vapor is much smaller than that of liquid, and hence the larger the bubble base length $\left(2 R_{b}\right)$, the lower the heat transfer efficiency is.

We also investigate the effects of surface superheating on the growing dynamics. The degree of surface superheating is defined by $\Delta T_{\text {sup }} \equiv T_{b}-T_{\mathrm{cx}}$. In our simulations, the coexistence temperature is fixed at $T_{\mathrm{cx}} \approx 0.84 T_{c}$ and the bottom-surface temperature $T_{b}$ is varied from $0.88 T_{c}$ to $0.93 T_{c}$. We plot in Fig. 4 the temporal evolution of the bubble base radius $R_{b}$ and that of the heat flux $Q_{b}$. We find that for sufficient superheating, the higher $\Delta T_{\text {sup }}$ is, the faster the bubble spreads laterally on the heating surface and, accordingly, the faster the heat transfer efficiency drops.

Finally, we plot in Fig. 5 the temporal evolution of the bubble area (volume in two dimensions), represented by an effective radius $R_{\text {eff }}$ defined by $\pi R_{\text {eff }}^{2} \equiv$ the instantaneous bubble area. It is seen that the rate of bubble growth is mainly

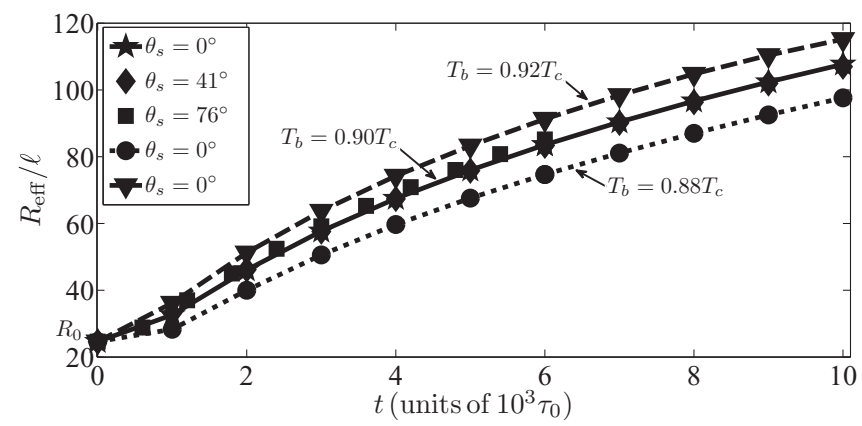

FIG. 5. Temporal evolution of the effective bubble radius $R_{\text {eff }}$ for different static contact angles and bottom-surface temperatures. Here $R_{\text {eff }}$ is defined by $\pi R_{\text {eff }}^{2}=$ the instantaneous bubble area (volume in two dimensions). determined by the degree of surface superheating with an extremely weak dependence on the surface wettability. This is because the liquid-to-vapor transition is localized around the contact line over a small length $\approx 3 \ell$ (see the upper panel of Fig. 2). The rate of the localized evaporation is mostly controlled by the degree of surface superheating with a weak dependence on the contact angle.

\section{B. Bubble departure under gravity}

Now we investigate the bubble dynamics on homogeneous surfaces under artificially large gravity. Starting from the equilibrium state of two-phase coexistence at temperature $T_{\mathrm{cx}} \approx 0.84 T_{c}$, we apply a gravity with $\mathcal{G}=10^{-4}$ and introduce a single bubble of initial radius $R_{0}=25 \ell$ on the bottom surface at $t=0$. The temperatures of the bottom and top surfaces are then changed to be $T_{b}=0.90 T_{c}$ and $T_{t}=0.775 T_{c}$, respectively. In Fig. 6, we plot the number density $n$, velocity $\mathbf{v}$, temperature $T$, and pressure $p$ for a representative state. Here the static contact angle is $\theta_{s}=60^{\circ}$. It is seen from the upper panel of Fig. 6 that the dynamic contact angle is a bit larger than its static value $\theta_{s}=60^{\circ}$. It is noted that a strong convective flow is present around the bubble, jointly induced by the gravity and the liquid-to-vapor transition near the contact line. In the nucleate boiling regime, heat transfer of high efficiency is facilitated, on the one hand, by the latent-heat adsorption of the growing bubble and, on the other hand, by the convective heat transfer accompanying the liquid circulation.

The middle panel of Fig. 6 shows that the temperature at the bubble surface is almost constant, close to $0.865 T_{c}$, which is actually a bit higher than $T_{\mathrm{cx}} \approx 0.84 T_{c}$. This is attributed to the pressure increase near the bubble due to the gravity. The lower panel of Fig. 6 shows the pressure profile along the $z$ direction, which consists of a few linear segments joined by fast variations in the liquid-vapor interfacial regions 

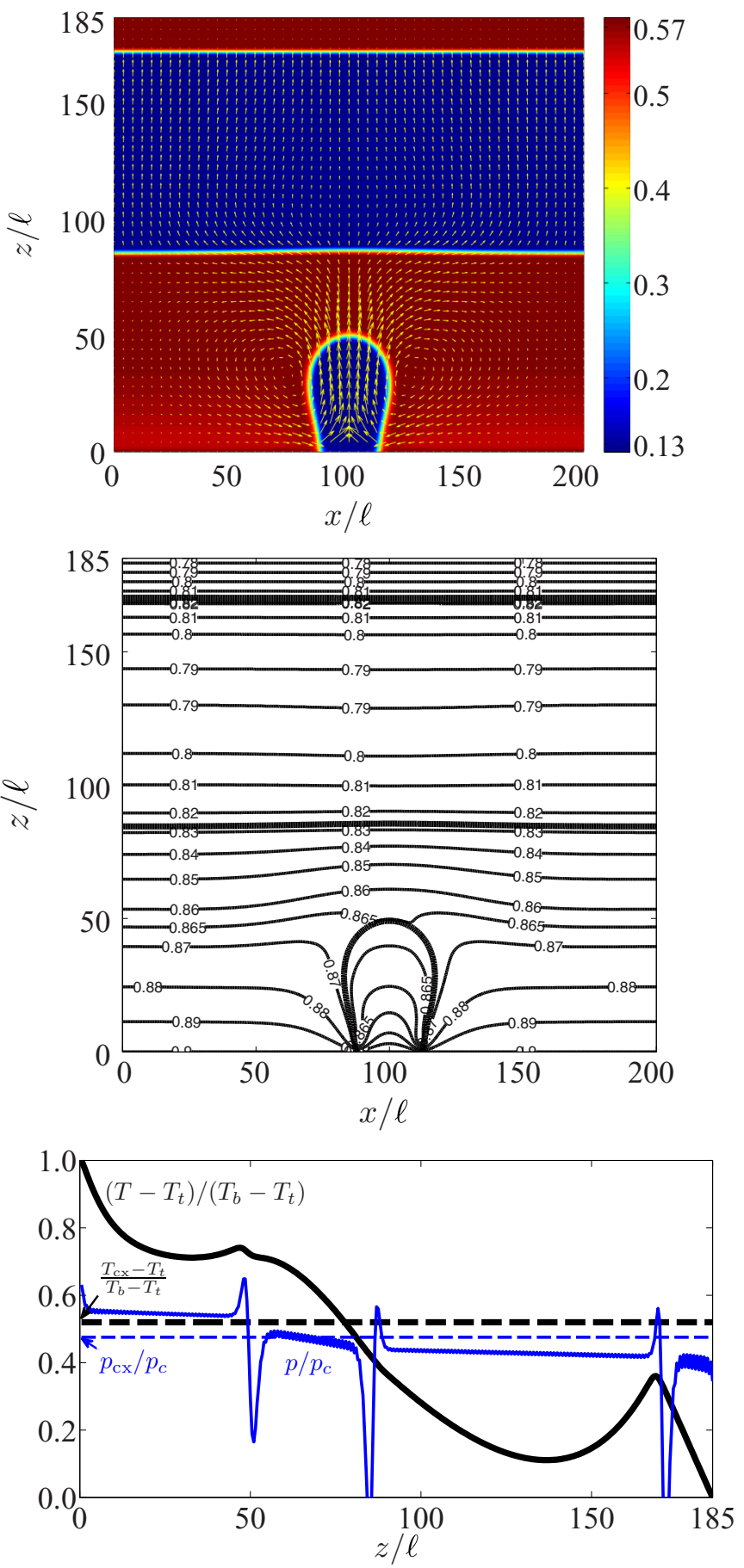

FIG. 6. (Color online) A bubble under gravity $\left(\mathcal{G}=10^{-4}\right)$ on a homogeneous surface with static contact angle $\theta_{s}=60^{\circ}$ and surface temperatures $T_{b}=0.90 T_{c}$ and $T_{t}=0.775 T_{c}$, recorded at time $t=3000 \tau_{0}$. The system measures $L_{x}=200 \ell$ by $L_{z}=185 \ell$ with $h_{l}=10 \ell, h_{v}=90 \ell$, and $H=85 \ell$ as defined in Fig. 1 . Upper panel: Density (color) and velocity (arrow) fields. Middle panel: Temperature contour (labeled thin lines) and liquid-vapor interfaces defined at $n=\left(n_{g}+n_{l}\right) / 2$ (thick lines). Temperature values are in units of $T_{c}$. Lower panel: Reduced pressure $p / p_{c}$ with $p$ calculated from Eq. (6) (thin solid blue line) and reduced temperature $(T-$ $\left.T_{t}\right) /\left(T_{b}-T_{t}\right)$ along the $z$ direction at $x=100 \ell$ (thick solid black line). The thin dashed blue line denotes the coexistence pressure $p_{\mathrm{cx}} \approx 0.48 p_{c}$, and the thick dashed black line denotes the coexistence temperature $T_{\mathrm{cx}} \approx 0.84 T_{c}$.
[30]. The pressure difference across the whole system along the $z$ direction is approximately $\langle n\rangle m g L_{z} \approx 0.20 p_{c}$ with the average density $\langle n\rangle \approx 0.38 / v_{0}$ and the system height $L_{z}=$ $185 \ell$. This further confirms that mechanical equilibrium is achieved quickly in this extremely nonisothermal system [49].

We then turn to the effects of surface wettability. We find that, similarly to the case of zero gravity, an increase in the static contact angle $\theta_{s}$ enhances the lateral bubble spreading on the heating surface. Accordingly, the residence time of the bubble on the surface becomes longer and the departure diameter of the bubble increases (see the insets of Fig. 7). This is attributed to the competition between lateral bubble spreading and vertical (buoyancy-induced) bubble deformation. The larger the bubble base length $\left(2 R_{b}\right)$, the stronger the bubble adhesion to the surface and hence the larger the bubble area (volume in two dimensions) required for departure. Therefore, higher hydrophobicity (i.e., larger contact angle) hinders the bubble departure and thus facilitates the transition from nucleate boiling into film boiling. This is consistent with the vapor recoil theory for the boiling transition $[5,13]$. Moreover, the insets of Fig. 7 indicate that there are two distinct regimes of bubble departure, depending on whether the departure is achieved by pinch-off.

Finally, we investigate the effects of surface superheating (see Fig. 8). We find that higher degree of surface superheating can also facilitate the lateral bubble spreading and thus delay the departure of the bubble from the heating surface.

\section{BUBBLE GROWTH AND DEPARTURE ON PATTERNED SURFACES}

The thermohydrodynamic simulations in the previous section demonstrate that, compared to hydrophobic surfaces, hydrophilic surfaces endow slower lateral spreading and smaller departure diameter of bubbles, which would lead to higher efficiency of heat transfer and hinder the transition from nucleate boiling into film boiling. In this respect, hydrophilic surfaces are superior to hydrophobic surfaces [1-3]. However, from the consideration of nucleation kinetics, hydrophobic surfaces possess much more active sites for bubble nucleation via thermal fluctuations under the same pressure and surface superheating [1-3]. For a given value of the contact angle $\theta_{s}$, the probability of a fluctuation producing a bubble nucleus on a solid surface is proportional to $e^{-\Delta \Omega_{s} / k_{B} T}$, where $\Delta \Omega_{s}=$ $\gamma \pi R_{0}^{2}\left(1+\cos \theta_{s}\right)^{2}\left(2-\cos \theta_{s}\right)$ is the surface free-energy cost for the nucleation of a bubble of radius $R_{0}$ and liquid-vapor interfacial tension $\gamma$ [39]. Therefore, the probability of bubble nucleation on hydrophobic surfaces $\left(\theta_{s}>90^{\circ}\right.$ and $\left.\cos \theta_{s}<0\right)$ is higher than that on hydrophilic surfaces $\left(\theta_{s}<90^{\circ}\right.$ and $\left.\cos \theta_{s}>0\right)$. In this section, we demonstrate that a designed patterned substrate may incorporate the advantages of both hydrophobic and hydrophilic surfaces. Below we present the bubble dynamics on a patterned (hydrophilic-hydrophobichydrophilic) surface in a two-dimensional geometry [see Fig. 1(b)].

\section{A. Stick-slip motion of contact line}

We first investigate the contact line motion in onecomponent fluids on patterned surfaces. Starting from the 

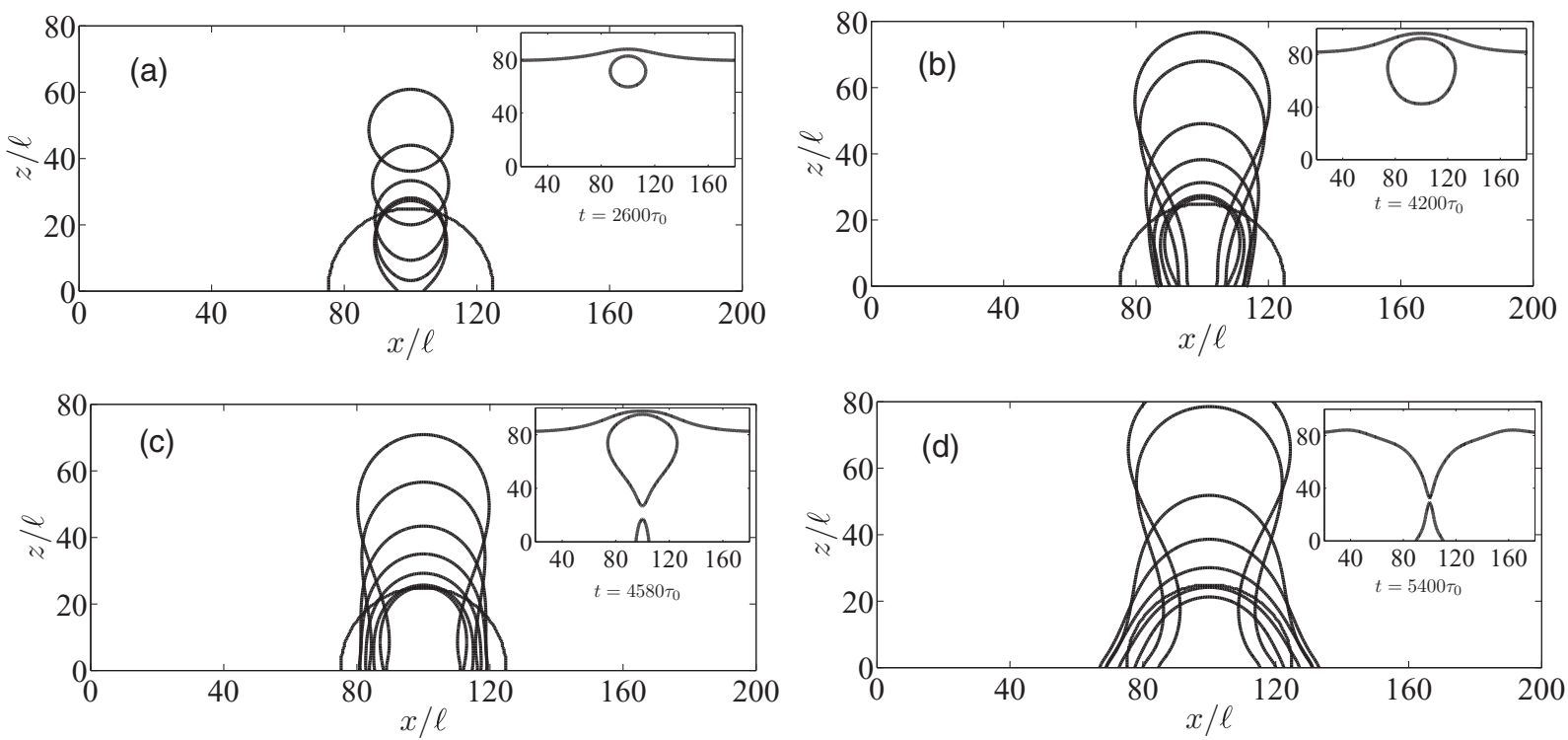

FIG. 7. Bubble departure under gravity $\left(\mathcal{G}=10^{-4}\right)$ from surfaces with different static contact angles: (a) $\theta_{s}=41^{\circ}$. Bubbles are plotted in sequence from $t=0$ to $2000 \tau_{0}$ with time interval $400 \tau_{0}$. (b) $\theta_{s}=60^{\circ}$. Bubbles are plotted in sequence from $t=0$ to $3600 \tau_{0}$ with time interval $600 \tau_{0}$ and at $t=3800 \tau_{0}$. (c) $\theta_{s}=76^{\circ}$. Bubbles are plotted in sequence from $t=0$ to $3600 \tau_{0}$ with time interval $600 \tau_{0}$ and at $t=4000 \tau_{0}$. (d) $\theta_{s}=101^{\circ}$. Bubbles are plotted in sequence from $t=0$ to $4800 \tau_{0}$ with time interval $800 \tau_{0}$ and at $t=5000 \tau_{0}$. Here the surface temperatures are $T_{b}=0.90 T_{c}$ and $T_{t}=0.775 T_{c}$. The system measures $L_{x}=200 \ell$ by $L_{z}=185 \ell$ with $h_{l}=10 \ell, h_{v}=90 \ell$, and $H=85 \ell$ as defined in Fig. 1 . Inset: Snapshots of bubbles that are detached from the surface and reaching the liquid-vapor interface originally at $z=H$ (see Fig. 1). In all four plots, a semicircular bubble of radius $R_{0}=25 \ell$ is introduced at the bottom surface at $t=0$. First, the bubble shrinks to a smaller one in a transient period ( $\sim$ hundreds of $\tau_{0}$ ) due to the mismatch between $R_{0}$ and the thermal and mechanical conditions around the bubble. Then the bubble grows continuously in both the horizontal and vertical directions. As the bubble radius becomes comparable with the capillary length $R_{c}(\approx 16 \ell$ here), the bubble evolution in the vertical direction becomes dominant over that in the horizontal direction due to buoyancy effect. Finally, the bubble detaches from the bottom surface.

equilibrium state of two-phase coexistence at temperature $T_{\mathrm{cx}} \approx 0.84 T_{c}$, we introduce a vapor bubble of $x$-extension $80 \ell$ sandwiched between liquids as shown in Fig. 9. The equilibrium state is illustrated in Fig. 9(a) and also shown in Fig. 9(b) at $t=0$. We then apply a body force along the $+x$ direction, whose magnitude equals the artificial gravity for $\mathcal{G}=10^{-4}$. Throughout the simulations, the temperatures of the bottom and top surfaces are both fixed at $T_{\mathrm{cx}} \approx 0.84 T_{c}$.

In Figs. 9(b) and 9(c), the contact line exhibits a stickslip motion. At the left hydrophobic-hydrophilic intersection $x=80 \ell$ [empty symbols in Fig. 9(c)], an abrupt increase of the contact-line velocity indicates a fast slip of the contact line when crossing that intersection. Near the right hydrophobic-hydrophilic intersection at $x=160 \ell$ [solid symbols in Fig. 9(c)], the vanishing contact-line velocity indicates the pinning of the contact line at that intersection. This kind of stick-slip motion also occurs in immiscible two-phase flows on patterned surfaces [50], where the contact line moves through boundary slip and bulk diffusion [41]. In one-component fluids, the contact line moves through boundary slip and liquid-vapor transition [32]. In both cases, the stick-slip motion is predominantly caused by the contact-angle switch at the
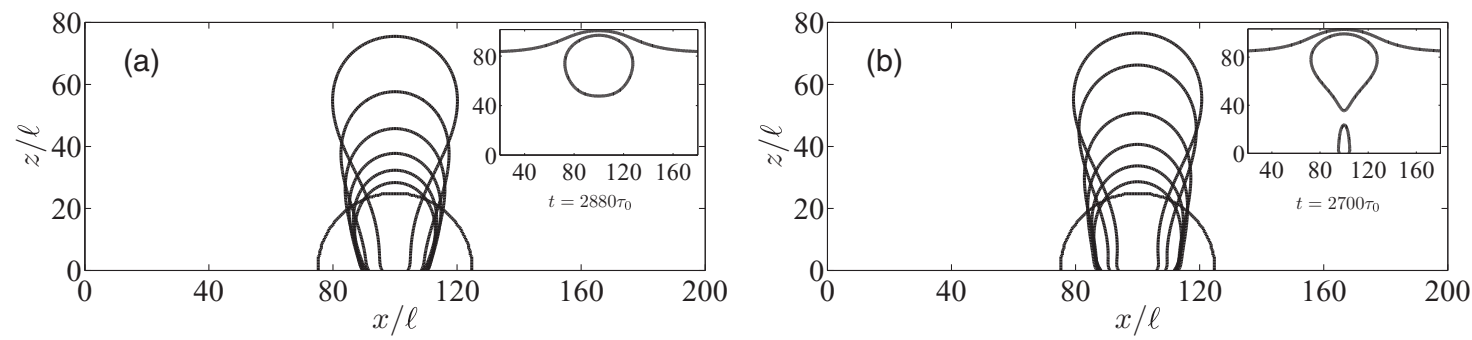

FIG. 8. Bubble departure under gravity $\left(\mathcal{G}=10^{-4}\right)$ from surfaces with different bottom-surface temperatures: (a) $T_{b}=0.92 T_{c}$. Bubbles are plotted in sequence from $t=0$ to $2400 \tau_{0}$ with time interval $400 \tau_{0}$. (b) $T_{b}=0.93 T_{c}$. Bubbles are plotted in sequence from $t=0$ to $2000 \tau_{0}$ with time interval $400 \tau_{0}$ and at $t=2200 \tau_{0}$. Here the static contact angle is $\theta_{s}=41^{\circ}$. The system measures $L_{x}=200 \ell$ by $L_{z}=185 \ell$ with $h_{l}=10 \ell$, $h_{v}=90 \ell$, and $H=85 \ell$ as defined in Fig. 1. Inset: Snapshots of bubbles that are detached from the surface and reaching the liquid-vapor interface originally at $z=H$ (see Fig. 1). The process of bubble growth followed by departure is similar to that described in the caption to Fig. 7. 

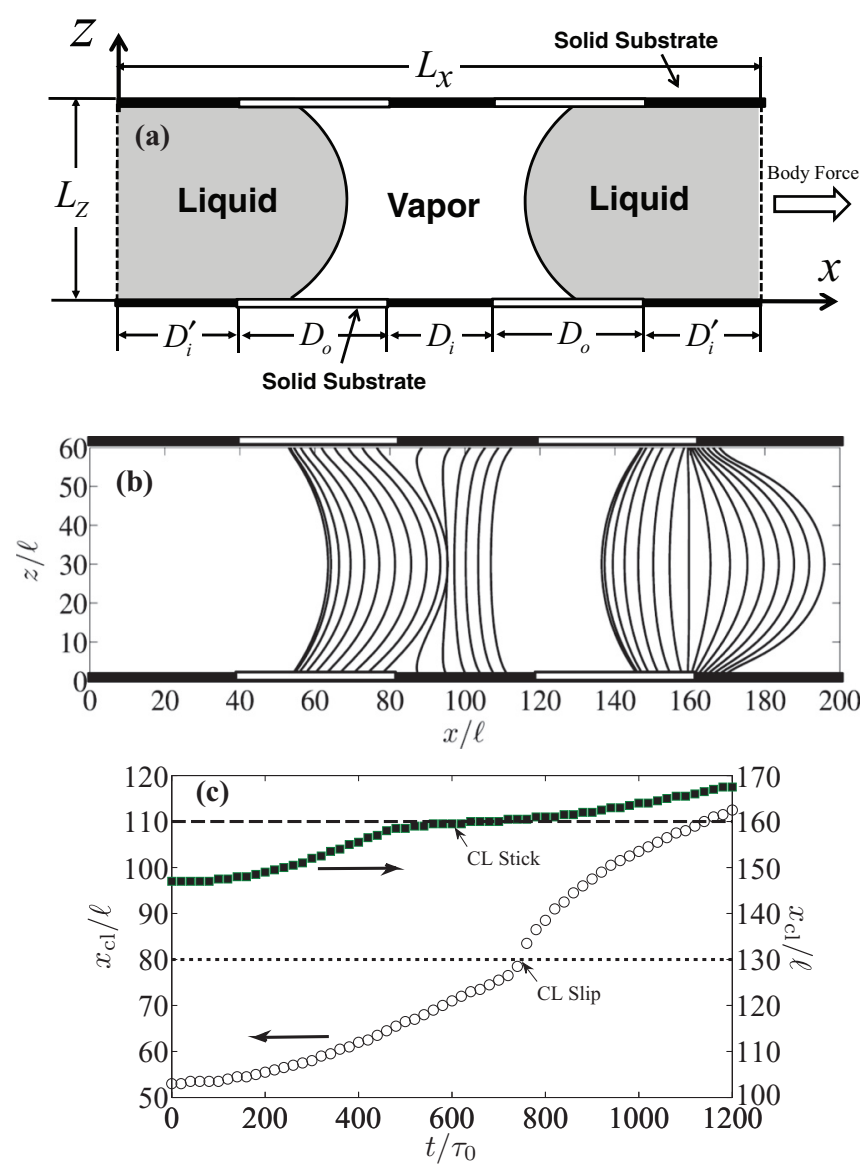

FIG. 9. (Color online) Stick-slip motion of contact line on chemically patterned surfaces. (a) Schematic illustration of the system. The black and white segments of the surface indicate the hydrophilic and the hydrophobic parts, respectively. The system is closed in the $x$ direction by the periodic boundary conditions. A body force is applied in the $+x$ direction to induce Poiseuille-type flow. (b) Temporal evolution of two liquid-vapor interfaces. The time ranges from $t=0$ (with the two interfaces denoted by two thick lines) to $1200 \tau_{0}$ with time step $\Delta t=80 \tau_{0}$. The system measures $L_{x}=200 \ell$ by $L_{z}=60 \ell$ with $D_{i}=D_{i}^{\prime}=D_{o}=40 \ell$. (c) Temporal evolution of the contact-line coordinates $x_{\mathrm{cl}}$ of the two liquid-vapor interfaces. The contact line of the left interface is represented by empty symbols and exhibits a fast slip near the intersection at $x=80 \ell$. The contact line of the right interface is represented by solid symbols and exhibits a pinning (stick) near the intersection at $x=160 \ell$.

intersections [50]. However, due to the participation of liquidvapor transition, the stick-slip motion in one-component fluids can be suppressed by surface superheating. This is attributed to the reduced contrast in contact angle, which decreases with the increasing degree of surface superheating [33,34,44]. It is interesting to note that stick-slip motion of the contact line also occurs in volatile nanoparticle suspensions and polymer solutions [51].

\section{B. Bubble growth under zero gravity}

Consider a bubble on a patterned (hydrophilichydrophobic-hydrophilic) surface under zero gravity. We first investigate the size effects of the hydrophobic segment on the growing dynamics of the bubble. The length $D_{o}$ of the hydrophobic segment is varied and the bubble evolution is displayed in Fig. 10. Since the capillary length $R_{c} \equiv\left(\gamma / n_{l} m g\right)^{1 / 2}$ tends to infinity under zero gravity, we are physically in the regime of $D_{o} \ll R_{c}$. We find that there are two distinct stages for bubble growth on the heating patterned surface. In the first stage, the bubble spreads laterally as fast as on a homogeneous hydrophobic surface [see Fig. 3(a4)] before the liquid-vapor interface reaches the boundary of the hydrophobic segment. As soon as the contact line arrives at the hydrophobic-hydrophilic intersection, it is pinned there, and, consequently, the bubble can only grow along the vertical direction. After a finite period of pinning, the contact line depins and the lateral bubble growth is continued on the hydrophilic segments.

We find that the wider the hydrophobic segment, the longer the pinning of the contact line is at the hydrophobichydrophilic intersection. Furthermore, an interesting separation of length scales and time scales is exhibited in Fig. 10(d). The bubble dynamics is affected by the hydrophobic segment only in the initial stage, characterized by a small length scale comparable with $D_{o}$. Later, the bubble growth becomes independent of the surface pattern, evidenced by the collapse of bubble profiles at a length scale much larger than $D_{o}$ [see Fig. 10(d)]. This corresponds to usual boiling on homogeneous surfaces, in which the nucleation sites (tiny pits or scratches of nano or micrometer scale) are much smaller than the capillary length $R_{c}$ (of millimeter scale). In this limit, the structural details of nucleation sites cannot be detected from the observation of long-time bubble dynamics and the measurement of heat transfer coefficient. We therefore conclude that hydrophilic surfaces patterned by nano- or microscale hydrophobic segments cannot significantly alter the bubble dynamics and improve the efficiency of heat transfer.

\section{Bubble departure under gravity}

Now we investigate the bubble dynamics on a patterned (hydrophilic-hydrophobic-hydrophilic) surface under artificially large gravity. Figure 11 shows the size effects of the hydrophobic segment by varying the length $D_{o}$. It is seen that the larger $D_{o}$, the longer the residence time of the bubble on the heating surface, and hence the larger the bubble departure diameter.

According to the above results and those in Sec. IV B for zero gravity, if the hydrophobic segment is too narrow (compared with the capillary length), then the hydrophobic pattern plays a negligible role and the surface just operates as a hydrophilic one. If the hydrophobic segment is too wide, then the departure diameter of the bubble is made very large, approaching what would be obtained on a hydrophobic surface. Combining these considerations, we conclude that under the operating condition of fixed degree of surface superheating $\Delta T_{\text {sup }} \equiv T_{b}-T_{\text {cx }}$, there exists an optimum length $D_{o}$ for the hydrophobic segment, which endows the patterned surfaces with abundant nucleation sites and smaller departure diameters simultaneously. In this sense, a patterned surface is able to incorporate the advantages of both hydrophobic and hydrophilic surfaces. The hydrophobic region provides abundant active sites for bubble nucleation while the surrounding hydrophilic region hinders the continuous lateral spreading of the bubble 

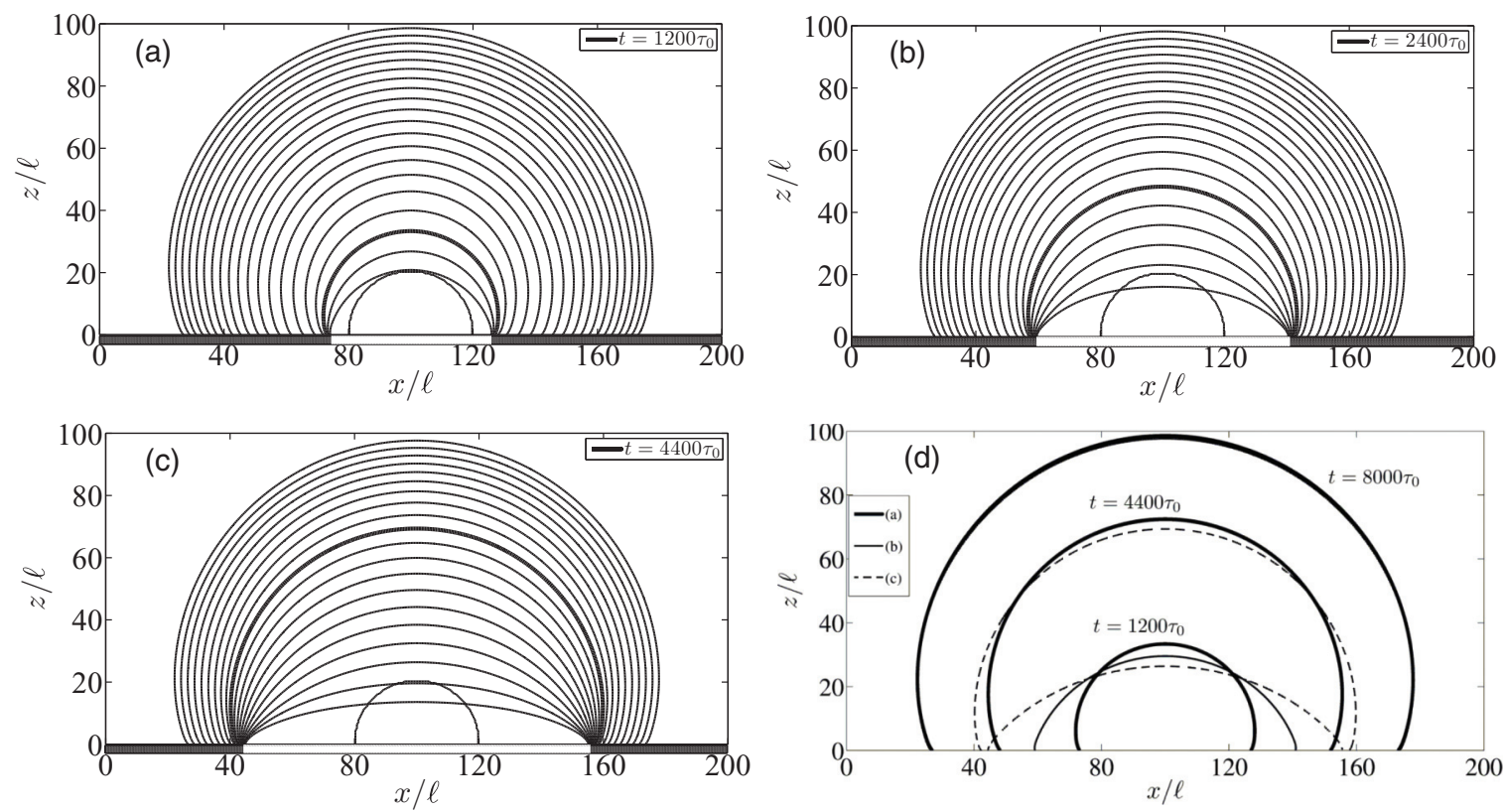

FIG. 10. Bubble growth under zero gravity on chemically patterned surfaces with different values of $D_{o}$ as defined in Fig. 1(b): (a) $D_{o}=50 \ell$, (b) $D_{o}=80 \ell$, and (c) $D_{o}=110 \ell$. The time step is $400 \tau_{0}$. The bold lines indicate the onset of the depinning of the contact line at the hydrophobic-hydrophilic intersection. (d) Bubble profiles selected from (a)-(c) with thick solid lines from (a), thin solid lines from (b), and thin dashed lines from (c). The collapse of bubble profiles at $t=8000 \tau_{0}$ indicates the separation of length and time scales. Here the static contact angle of the hydrophilic segment is $\theta_{s}=0^{\circ}$ and that of the hydrophobic segment is $\theta_{s}=180^{\circ}$. The surface temperatures are $T_{b}=0.90 T_{c}$ and $T_{t}=0.775 T_{c}$. The system measures $L_{x}=200 \ell$ by $L_{z}=280 \ell$ with $h_{l}=10 \ell, h_{v}=120 \ell$, and $H=150 \ell$ as defined in Fig. 1 .

by pinning the contact line at the hydrophobic-hydrophilic intersection. The former increases the density of nucleated bubbles while the latter results in a small bubble departure diameter and helps prevent the transition from nucleate boiling into film boiling. These effects would jointly enhance the efficiency of heat transfer. We would like to emphasize that our conclusion is actually qualitatively consistent with recent experimental measurement of heat transfer coefficients on various homogenous and chemically patterned surfaces [37]. It has been experimentally found that the largest heat transfer coefficients are achieved not on surfaces with spatially uniform wettability but on heterogeneous surfaces textured with hydrophilic and hydrophobic regions [37,52-54].

To make our study more complete, numerical simulations are also carried out for bubble dynamics on a patterned (hydrophobic-hydrophilic-hydrophobic) surface-a hydrophobic surface decorated with a hydrophilic segment in the center. Under zero gravity, first the bubble grows slowly as on a homogeneous hydrophilic surface. As soon as the contact line arrives at the hydrophilic-hydrophobic intersection, it slips
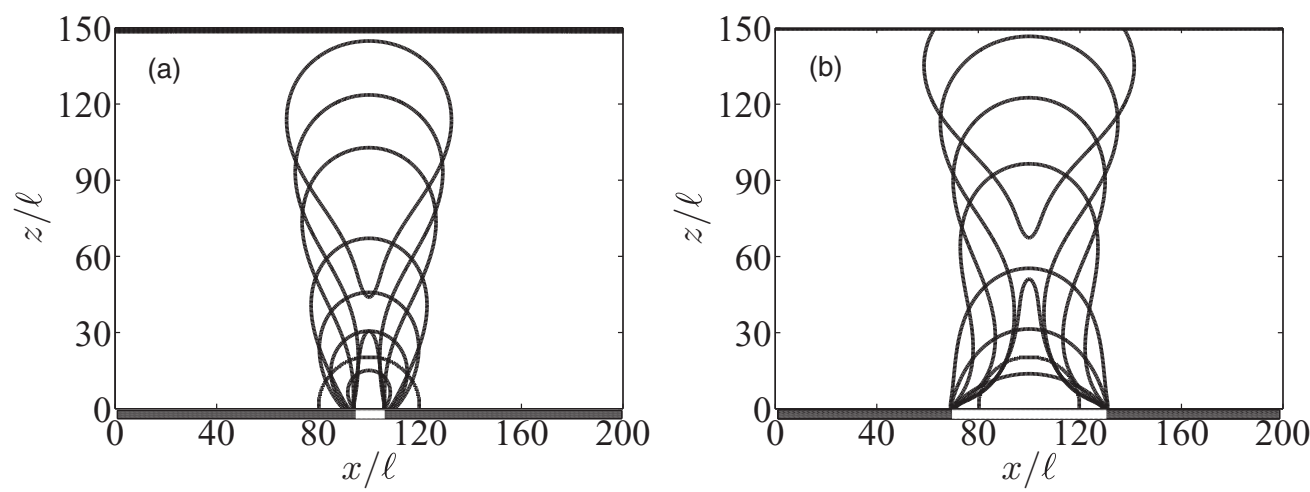

FIG. 11. Bubble departure under gravity $\left(\mathcal{G}=5.0 \times 10^{-5}\right)$ on chemically patterned surfaces with different values of $D_{o}$ as defined in Fig. 1: (a) $D_{o}=10 \ell$. Bubbles are plotted in sequence at $t=0,1000 \tau_{0}, 3000 \tau_{0}, 4000 \tau_{0}, 5000 \tau_{0}, 6000 \tau_{0}, 6400 \tau_{0}$, and 6760 $\tau_{0}$. (b) $D_{o}=60 \ell$. Bubbles are plotted in sequence at $t=0,1000 \tau_{0}, 3000 \tau_{0}, 5000 \tau_{0}, 7000 \tau_{0}, 7600 \tau_{0}, 8000 \tau_{0}$, and $8360 \tau_{0}$. Here the static contact angle of the hydrophilic segment is $\theta_{s}=0^{\circ}$ and that of the hydrophobic segment is $\theta_{s}=180^{\circ}$. The surface temperatures are $T_{b}=0.90 T_{c}$ and $T_{t}=0.775 T_{c}$. The system measures $L_{x}=200 \ell$ by $L_{z}=250 \ell$ with $h_{l}=10 \ell, h_{v}=90 \ell$, and $H=150 \ell$ as defined in Fig. 1 . The process of bubble growth followed by departure is similar to that described in the caption to Fig. 7. 
quickly across the intersection. Then the bubble spreads as on a homogeneous hydrophobic surface. Under artificially large gravity, if the hydrophilic segment is too narrow compared with the capillary length, then its effect is negligible and the whole surface just operates as a hydrophobic one with a low rate of heat transfer. If the hydrophilic segment is wide enough compared with the capillary length, then the patterned surface just operates as a hydrophilic one with a higher rate of heat transfer. Therefore, for a bubble growing on a hydrophobic surface decorated with a hydrophilic segment, there is a minimum length $D_{i}$ of hydrophilic segment to achieve a heat transfer rate appreciably higher than that on a homogeneous hydrophobic surface. Combining the above results with those for a hydrophilic surface decorated with a hydrophobic segment, we conclude that there exists a minimum length $D_{i}$ for hydrophilic segment and an optimal length $D_{o}$ for hydrophobic segment. In fact, both $D_{i}$ and $D_{o}$ should be of the same order of magnitude of the capillary length. Therefore, the aspect ratio of the hydrophobic patch on hydrophilic surface, defined by $D_{o} /\left(D_{o}+D_{i}\right)$, should be about 0.5 in order to achieve a high rate of heat transfer. Moreover, it can be predicted that in a three-dimensional situation, the length scale in each direction on the patterned surface should be comparable to the capillary length with the aspect ratio close to 0.5 [37].

\section{CONCLUDING REMARKS}

We have investigated the thermohydrodynamics of a single bubble in pool boiling of one-component fluids. We employed the DVDWT as an efficient method based on the diffuseinterface modeling of liquid-vapor flows involving liquidvapor transition in a nonuniform temperature field [30,32]. The DVDWT describes the various physical components that are involved and interrelated in the pool boiling, including two-phase hydrodynamics, liquid-vapor transition, latent-heat transport, and wetting dynamics. We carried out simulations in two spatial dimensions for bubbles on heating surfaces under zero and artificially large gravity, respectively. Simulations for zero gravity are motivated by the initial stage of bubble growth in nucleate boiling, during which the bubble size is too small for the gravity to play an appreciable role. On the other hand, simulations for artificially large gravity are numerical demonstrations of the buoyancy-induced bubble departure under Earth's gravity.

The focus of the present work is on the effects of surface wettability upon the bubble dynamics. For this purpose, we first studied bubbles on homogeneous substrates. Our simulations show that under zero gravity, the speed of lateral bubble spreading increases with increasing contact angle. Consistent with this finding, our simulations also show that under gravity, the diameter of bubble departure increases with increasing contact angle. Physically, the larger the bubble base length, the stronger the bubble adhesion to the substrate, and hence the larger the bubble volume is required for departure. We also investigate the effects of surface superheating on the bubble dynamics with similar findings that can be interpreted using the temperature dependence of contact angle [13,34,44].
We then studied the bubble dynamics on patterned (hydrophilic-hydrophobic-hydrophilic) substrates. Our simulations show that under zero gravity, the larger the hydrophobic segment, the longer the pinning of the contact line at the hydrophobic-hydrophilic intersection. Consistently, our simulations also show that under gravity, the larger $D_{o}$ is, the longer the residence time of the bubble on the heating surface, and hence the larger the bubble departure diameter. We thereby conclude that there exists an optimum length $D_{o}$ of the hydrophobic segment, which endows the patterned surface with abundant nucleation sites (on the hydrophobic segment) and smaller departure diameters (constrained by the contact line pinning at the hydrophobic-hydrophilic intersection). Physically, the optimum value for $D_{o}$ is expected to be comparable with the capillary length $R_{c}$. In this sense, a hydrophilic surface patterned with hydrophobic patches of length $D_{o}$ is able to incorporate the advantages of both hydrophobic and hydrophilic surfaces. This conclusion from our simulations is in agreement with recent experimental observations [37,52-54].

We would like to point out that the contact-line pinning mentioned above is part of the stick-slip motion of contact line on patterned surfaces. Our simulations have demonstrated that the stick-slip motion in one-component fluids is similar to that in immiscible binary mixtures, both caused by the switch of contact angle across the hydrophobic-hydrophilic intersection [50]. In one-component fluids, however, the stickslip motion can be suppressed by surface superheating due to the temperature dependence of the dynamic contact angle $[33,34,44]$.

Finally, we make a few remarks.

(i) Since the mesh size $\Delta x=\Delta z=0.5 \ell$ used to resolve the diffuse interface is only a few angstroms, our simulated systems are actually very small, up to hundreds of nanometers at most. Moreover, an artificially large gravity is introduced to make the capillary length $R_{c}$ sufficiently small and thus accessible in our simulations. It is our expectation that a larger scale simulation of pool boiling using the DVDWT will soon be computationally achievable. This will surely contribute to the study of the fundamental physical aspects of boiling.

(ii) In this work, the wettability of the heating surface is solely modeled by short-range interactions at the fluid-solid interface. The long-range van der Waals interaction has not been taken into account, which is essential to the physical understanding of wetting phenomena [55,56].

(iii) The present work demonstrates that boiling heat transfer can be enhanced by patterning hydrophilic surfaces with hydrophobic patches of size comparable with the capillary length. However, the contrast in contact angle, which is necessitated by the effectiveness of such a design, can be significantly reduced by surface superheating $[33,34,44]$. We further propose that more effective surface can be engineered by combining geometric pattern with chemical pattern [1-3].

(iv) The present work deals with the dynamics of a single bubble. However, hydrodynamic interactions between bubbles must play important roles, particularly in the layer of crowded bubbles near the heating surface [1-3]. It has been predicted that through hydrodynamic interactions, bubbles can even detach from the heating surface under zero gravity [4]. 
(v) The DVDWT can also be applied to investigate the bubble dynamics in many other boiling scenarios, e.g., saturated flow boiling and subcooled (pool and flow) boiling [1-3]. Particularly, in subcooled pool boiling, the liquid near the heating surface is superheated while the liquid in the bulk is undersaturated, i.e., subcooled. Typically, vapor bubbles will be formed and grow near the surface. As the bubbles become large enough, they detach from the surface. During their buoyancy-induced rise, they shrink and collapse rapidly in the subcooled liquid, signaled by audible sound emission. The rapid dynamics of bubble collapse coupled with sound emission and propagation, which is not yet well understood, can be studied by using the DVDWT. (vi) The pool boiling of binary mixtures may also be explored by following the general framework of the DVDWT. In binary mixtures, even at small solute concentrations, the fluid-fluid interface is nonisothermal and therefore the surface Marangoni force may play an essential role [57].

\section{ACKNOWLEDGMENTS}

This publication is based on work partially supported by Award No. SA-C0040/UK-C0016 from the King Abdullah University of Science and Technology (KAUST) and Hong Kong RGC Grant No. 604013.
[1] L. S. Tong and Y. S. Tang, Boiling Heat Transfer and Two-Phase Flow (Taylor \& Francis, Washington, D.C., 1997).

[2] V. P. Carey, Liquid-Vapor Phase-Change Phenomena (Taylor \& Francis, New York, 2008).

[3] V. K. Dhir, Annu. Rev. Fluid Mech. 30, 365 (1998).

[4] Yu. A. Buyevich, Int. J. Fluid Mech. Res. 26, 189 (1999).

[5] V. S. Nikolayev and D. A. Beysens, Europhys. Lett. 47, 345 (1999).

[6] V. K. Dhir, Am. Inst. Chem. Eng. J. 47, 813 (2001).

[7] H. K. Forster and N. Zuber, J. Appl. Phys. 25, 474 (1954).

[8] V. K. Dhir, Int. J. Heat Fluid Flow 12, 290 (1991).

[9] J. Straub, Int. J. Therm. Sci. 39, 490 (2000).

[10] A. Onuki, Phase Transition Dynamics (Cambridge University Press, Cambridge, 2002).

[11] Y. Garrabos, C. Lecoutre-Chabot, J. Hegseth, V. S. Nikolayev, D. Beysens, and J. P. Delville, Phys. Rev. E 64, 051602 (2001).

[12] V. Nikolayev, D. Beysens, and D. Chatain, arXiv:0707.3009.

[13] V. S. Nikolayev, D. Chatain, Y. Garrabos, and D. Beysens, Phys. Rev. Lett. 97, 184503 (2006).

[14] S. R. de Groot and P. Mazur, Non-Equilibrium Thermodynamics (Dover, New York, 1984).

[15] L. D. Landau and E. M. Lifshitz, Fluid Mechanics (Pergamon Press, Oxford, 1987).

[16] S. Osher and J. A. Sethian, J. Comput. Phys. 79, 12 (1988).

[17] G. Tomar, G. Biswas, A. Sharma, and A. Agrawal, Phys. Fluids 17, 112103 (2005).

[18] X. Shan and H. Chen, Phys. Rev. E 47, 1815 (1993).

[19] A. J. Briant, A. J. Wagner, and J. M. Yeomans, Phys. Rev. E 69, 031602 (2004)

[20] M. Gross, F. Varnik, D. Raabe, and I. Steinbach, Phys. Rev. E 81, 051606 (2010).

[21] T. Seta and K. Okui, J. Therm. Sci. Tech. 1, 125 (2006).

[22] A. Márkus and G. Házi, Nucl. Eng. Des. 248, 238 (2012).

[23] S. Ryu and S. Ko, Nucl. Eng. Des. 248, 248 (2012).

[24] H. Inaoka and N. Ito, Physica A 392, 3863 (2013).

[25] H. Okumura and N. Ito, Phys. Rev. E 67, 045301 (2003).

[26] H. Watanabe, M. Suzuki, and N. Ito, Phys. Rev. E 82, 051604 (2010).

[27] D. T. Semiromi and A. R. Azimian, Heat Mass Transfer 48, 141 (2012).

[28] C. Wu, X. Xu, and T. Qian, J. Phys.: Condens. Matter 25, 195103 (2013).
[29] A. Onuki, Phys. Rev. Lett. 94, 054501 (2005).

[30] A. Onuki, Phys. Rev. E 75, 036304 (2007).

[31] X. Xu, C. Liu, and T. Qian, Commun. Math. Sci. 10, 1027 (2012).

[32] X. Xu and T. Qian, J. Chem. Phys. 133, 204704 (2010).

[33] X. Xu and T. Qian, Phys. Rev. E 85, 051601 (2012).

[34] X. Xu and T. Qian, Phys. Rev. E 85, 061603 (2012).

[35] X. Xu and T. Qian, Phys. Rev. E 87, 043013 (2013).

[36] T. Laurila, A. Carlson, M. Do-Quang, T. Ala-Nissila, and G. Amberg, Phys. Rev. E 85, 026320 (2012).

[37] A. R. Betz, J. Jenkins, C.-J. Kim, and D. Attinger, Int. J. Heat Mass Transfer 57, 733 (2013).

[38] R. Teshigawara and A. Onuki, Phys. Rev. E 82, 021603 (2010).

[39] L. D. Landau and E. M. Lifshitz, Statistical Physics, Part 1 (Pergamon Press, Oxford, 1980).

[40] J. Koplik, J. R. Banavar, and J. F. Willemsen, Phys. Rev. Lett. 60, 1282 (1988).

[41] T. Qian, X.-P. Wang, and P. Sheng, Phys. Rev. E 68, 016306 (2003).

[42] J.-L. Barrat and F. Chiaruttini, Mol. Phys. 101, 1605 (2003).

[43] Z. Ge, D. G. Cahill, and P. V. Braun, Phys. Rev. Lett. 96, 186101 (2006).

[44] R. Teshigawara and A. Onuki, Europhys. Lett. 84, 36003 (2008).

[45] J. W. Cahn, J. Chem. Phys. 66, 3667 (1977).

[46] M. S. Plesset and A. Prosperetti, Annu. Rev. Fluid Mech. 9, 145 (1977).

[47] W.-J. Yang and H.-C. Yeh, Am. Inst. Chem. Eng. J. 12, 927 (1966).

[48] Y. Nam, J. Wu, G. Warrier, and Y. S. Ju, J. Heat Transfer 131, 121004 (2009).

[49] R. Hołyst and M. Litniewski, Phys. Rev. Lett. 100, 055701 (2008).

[50] X.-P. Wang, T. Qian, and P. Sheng, J. Fluid Mech. 605, 59 (2008).

[51] L. Frastia, A. J. Archer, and U. Thiele, Phys. Rev. Lett. 106, 077801 (2011).

[52] A. Betz, J. Xu, H. Qiu, and D. Attinger, Appl. Phys. Lett. 97, 141909 (2010).

[53] H. J. Jo, H. Kim, H. S. Ahn, S. Kang, J. Kim, J. S. Shin, and M. H. Kim, Trans. Korean Soc. Mech. Eng. B 34, 655 (2010).

[54] H. Jo, H. S. Ahn, S. Kang, and M. H. Kim, Int. J. Heat Mass Transfer 54, 5643 (2011).

[55] P. G. de Gennes, Rev. Mod. Phys. 57, 827 (1985).

[56] Y. Di and X.-P. Wang, J. Comput. Phys. 228, 1380 (2009).

[57] A. Onuki, Phys. Rev. E 79, 046311 (2009). 